\title{
A Modified Successive-Cancellation Stack Polar Decoder
}

\author{
Chun $\mathrm{Li}^{1, \mathrm{a}}$, Xinhai Tong ${ }^{2, \mathrm{~b}}$ \\ ${ }^{1}$ PLA University of Science and Technology, Nanjing, China \\ ${ }^{2}$ PLA University of Science and Technology, Nanjing, China \\ alc19891989@126.com, btongxinhai@sohu.com,
}

Keywords: Algorithm reformulation, Stack decoding, Polar codes, Successive cancellation (SC).

\begin{abstract}
Polar codes represent an emerging correcting codes with power to approach the capacity of a discrete memoryless channel. However, the decoding performance of polar codes with traditional successive cancellation (SC) algorithm cannot match that of the low-density parity-check or Turbo codes. In this paper, a modified successive cancellation stack (MSCS) polar decoder is proposed. Unlike the original SC stack polar (SCS) decoders, we propose a modified path that can significantly reduce latency of SCS decoders. Simulation results in the binary-input additive white Gaussian noise channel show that modified algorithm has the slightly performance as the SCS algorithm. Moreover, the time and space complexity of the MSCS decoder is much lower than that of the SCS and can be very close to that of the SC in the high SNR regime.
\end{abstract}

\section{Introduction}

In recent years, with the continuous development of coding theory in digital communications, the polar codes have been proven to achieve the symmetric capacity of any binary-input discrete memoryless channel (B-DMC) [1]. Channel polarization, a concept central to polar codes, is performed by channel splitting and channel combining operation on independent copies of a given B-DMC. However, the finite-length performance is unsatisfying. So in order to improve the performance of polar codes, many decoding algorithms have been proposed [2]-[4]. Belief propagation (BP) [3],[4] and linear programming (LP) [5] decoders are reported to have a significant improvement over SC. Later, successive cancellation stack (SCS) was presented in[6]. Simulation results show that polar codes with the use of SCS algorithm combined with simple cyclic redundancy check (CRC) and systematic encoding methods can outperform the same length and rate LDPC codes[7]. As a result, the SCS algorithm is believed to be the key for decoding of polar codes to be applicable in practical systems.

Performance and complexity analysis based on simulations shows that under proper configuration, SC list and SCS decoding algorithms can approach the performance of the maximum likelihood (ML) decoding but with acceptable complexity. The time complexity of the SCS decoding decreases with the increase of the signal-to noise (SNR) and can be very close to that of the SC decoding in the high SNR regime. Compared with SCL, it has a much lower time complexity.

Under this situation, the contribution of this paper include the following several aspects. First, the SCS decoding algorithm is described under a unified manner of a path search procedure on the code tree. Then, by combining the principle of SCS, a modified algorithm is proposed. This proposed algorithm is a generic SC decoding scheme and can reduce the complexity.

\section{Successive Cancellation Stack Algorithm}

A code tree is used to describe the decoding process of polar code. In this code tree, except for the leaf nodes and the frozen nodes, each node has two descendants and the corresponding branches are labeled with 0 and 1, respectively. The SC decoding of polar codes can be regarded as a greedy search algorithm over the connection stage code tree. Fig.1 gives a simple example. Between the two branches associated with an information bit at a certain level, only the one with the larger 
probability is selected for further processing. However once a bit is wrongly decided, there is no chance to correct it in the future decoding procedure.

In contrast, the SCS decoder always searches along the best candidate path in the stack and the candidates no longer have the same length. Fig.2 gives an example of a code tree with four levels. Whenever the top path in the stack that has the largest path metric reaches a leaf node, the decoding process stops and outputs this path. Different with the SCL algorithm, the candidates in the stack of SCS have different length. These paths are sorted by path metrics in descending order, where different length means the number of path extensions can be reduced.

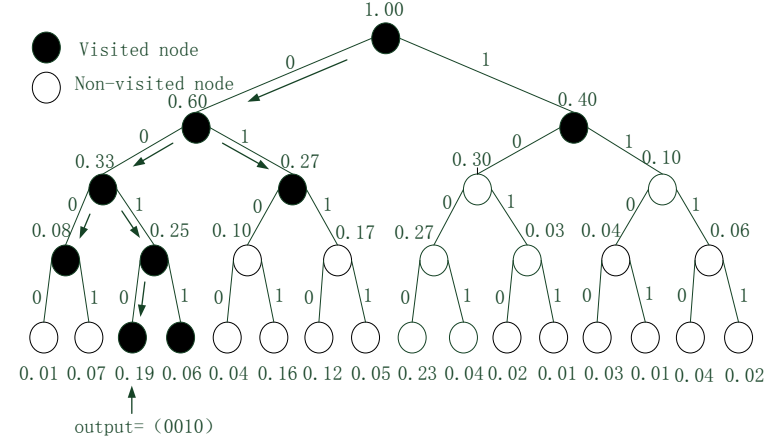

Fig. 1. Searching process of SC decoder

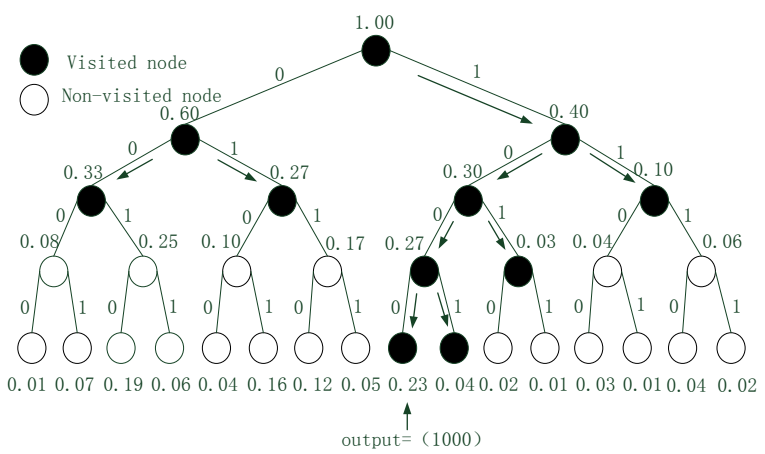

Fig. 2. Searching process of SCS decoder

Initially, the metric for null path $\phi$ is $M(\phi)=0$. The updated rule of the metric is: when the extended bit is a frozen bit, the metric remains fixed:

$$
M\left(d_{1}^{i}\right)=M\left(d_{1}^{i-1}\right), \quad \mathrm{i} \in \mathrm{A}^{c}
$$

Otherwise, if the extended bit is send message, the path metric could be calculated as follow formula:

$$
M\left(d_{1}^{i}\right)=\log W_{N}^{(i)}\left(y_{1}^{N}, d_{1}^{i-1} \mid d_{i}\right), \quad \mathrm{i} \in \mathrm{A}
$$

\section{Modified Successive Cancellation Algorithm}

In the MSCS decoder, the bits in the path are just tentative values and probabilities of a certain information bit that could be used for further decoding. Let $\mathrm{D}$ and $\mathrm{T}$ be the maximal and instantaneous depth of the stack, so we can get the modified successive cancellation stack algorithm as follows:

1. Input: Likelihood of each bit in the received codeword.

2. Initialization: Stack depth $\mathrm{T}=0$ and compute the root node of code tree.

3. Push null path into stack and $\mathrm{T}=\mathrm{T}+1$.

4. Pop the path $d_{1}^{i}$ from top stack and $\mathrm{T}=\mathrm{T}-1$.

5. If $u_{i+1} \in A^{c}$ the current path $d_{i+1}=0$ otherwise extend $\left(d_{1}^{i}, 0\right)$ and $\left(d_{1}^{i}, 1\right)$ then calculate the metric by (1) and (2).

6. Push: if $u_{i+1}$ is information bit and $T>D-2$, delete the bottom of the stack then set $T=T-1$ and push the two extended path into the stack then set $T=T+2$ otherwise $u_{i+1}$ is frozen bit push $\left(d_{1}^{i}, 0\right)$ into the stack and set $T=T+1$

7. Judge: if $i \triangleq N / 2$ reduce the depth of stack $\mathrm{D}$ and $\mathrm{T} \quad D=$ floor $(D / 2) \mathrm{T}=$ floor(T/2)

8. Sort: rearrange paths in descending metrics

9. Decision: if the top path reach the leaf node output the top path as the decision otherwise go to 4

A direct implementation of the SC decoder will require $O(N \log N)$ computations. As for the implementation aspect, a space-efficient structure implement the SCL decoder, the time and space complexities are $O(L N \log N)$. Different with SCL decoder, MSCS decoder could reduce the unnecessary path copy operations and memory occupation. 


\section{Simulation Result and Analysis}

Fig. 3 gives the BER performance comparison of different decoding algorithms of polar code the AWGN channel. The list size of the SCL decoder is fixed to $\mathrm{L}=8$. The maximal depth of the stack in the SCS decoder is $\mathrm{D}=50$. We can see that the performance of MSCS algorithm is better than the SC algorithm. To analyse the complexity of the decoders, the MSCS decoder is variable and decreases with the increasing SNR. Compared with the SCS, MSCS can save a large number of unnecessary computations especially when working in the high SNR regime. The stack used in SCS consumes a much larger space than the stack used in MSCS. However, the most appropriate value of D is dependent on the specific SNR and is difficult to determine.

All of above analytical result indicate the performance of MSCS decoding will be reduced complexity by get rid of little value paths. The MSCS decoder has very low time complexity and its performance is very close to that of the SCS decoder. Hence, it can achieve better trade-off between complexity and performance than other decoding algorithms.

\section{Conclusion}

In this paper, a modified SCS polar decoder was proposed by get rid of paths of the decoder. The reformulated algorithm can reduce the latency significantly with performance loss. For these reasons, polar code under MSCS decoding can be a competitive candidate in future communication systems. Finally, one important thing should be noted that, in this work, a particular method is introduced to reduce the complexity, while the how to choose appropriate $\mathrm{D}$ is still needed to be further investigated.

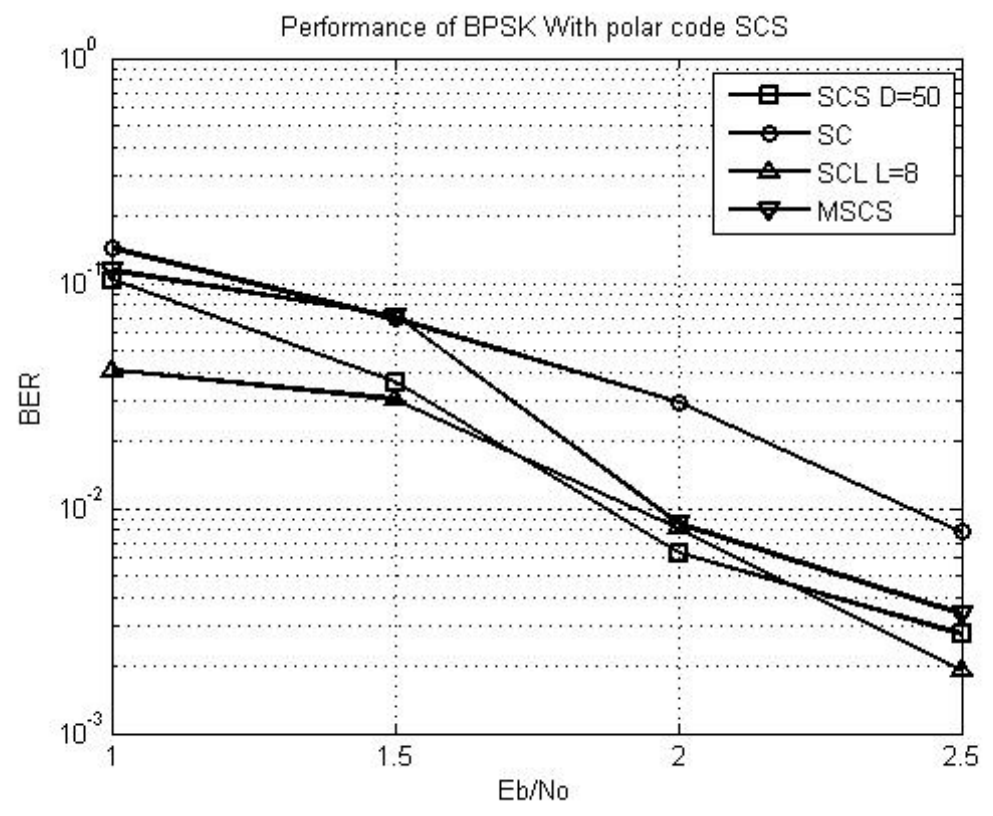

Fig. 3. Performance comparison of SC,SCL,SCS,MSCS algorithms with the $(256,128)$ polar code under BI-AWGN channel

\section{References}

[1] E. Arikan, "Channel polarization: A method for constructing capacity achieving codes for symmetric binary- input memoryless channels.” IEEE Trans. Inf. Theory. Vol. 55, no.7, pp. 3051-3073, Jul. 2009.

[2] I. Tal and A. Vard, “List Decoding of Polar codes,” in Proc. IEEE Int. Symp. Inf. Theory, 2011. pp. 1-5.

[3] Hussami, N. Korada, S.B., and Urbanke,R.: "Performance of polar codes for channel and source coding” Proc. of IEEE Int. Symp. On Information Theory, Seoul, South Korea. July 2009. 
[4] Jelinek, F. “A fast sequential decoding algorithm using a stack”, IBM. J. Res. Dev., 1969,13,pp.675-685.

[5] Goela, N., Korada, S.B. and Gastpar. M.: “On LP decoding of polar codes”. Information Theory Workshop (ITW) ,August-September 2010.

[6] K. Niu and K. Chen, “Stack Decoding of Polar Codes,” Election. Letter, vol. 48, no. 12, June 2012, pp. 695-696.

[7] K. Niu and K. Chen, “CRC-Aided Decoding of Polar Codes,” IEEE Communication Letter, vol. 16, no. 10, Oct. 2012, pp. 1668-1671. 